\title{
Mono-Microbial Diabetic Foot infections: Feasibility for Prompt Treatment
}

\author{
C. Meenakshi Sundaram*, G.Jayalakshmi and P.Selvaraj
}

Sri Venkateswara Medical College Hospital \& Research Institute, Puducherry, India

*Corresponding author

\section{A B S T R A C T}

\begin{tabular}{|l|}
\hline Ke y w o r d s \\
Diabetic foot \\
ulcers-Mono- \\
microbial \\
infections- \\
Amikacin -a \\
promising drug
\end{tabular}

\begin{abstract}
A detailed analysis of retrospective data on bacterial culture and sensitivity documented in a tertiary care hospital in Puducherry was carried out, with an aim of assessing the feasibility of giving prompt treatment topatients with mono-microbialdiabetic foot ulcer infections. In a patient strength of 40 , consisting of 30 males and 10 females,mono-microbial infection rate was $45.0 \%$ (18/40), andpolymicrobialinfection was $55.0 \%$ (22/40). About $55.5 \%$ of patients affected by mono-microbial infection were in the age group of 50 to 69 years, among whom, males formed a majority. Infection by a single pathogen was caused by 5-species of Gram-positive (lone) pathogens(namely, Staphylococcus aureus or MRSA,or CONS, or Enterococcus species, or Streptococcus species), affecting 10 persons; and by 7-species of Gram-negative (lone) pathogens ( namely, Pseudomonas species, or Pseudomonas aeruginosa, or Proteus species or Escherichia coli or Klebsiellaoxytoca). This is in contrast to the experiences of Western countries where mono--microbial diabetic foot infections are mostly reported to be caused by Gram-positive pathogens, whereas, Gram-negative pathogens are reported to be associated with mixed-flora of poly-microbial infections. The bacterial sensitivity data indicated that Amikacin was a promising drug for empirical therapy against all Gram-negative organisms involved, in mono-microbial infections, excepting Proteus vulgaris, against which Imipenem, or Levofloxacin was found to be effective. Amikacin was also effective against the three Gram-positive pathogens, namely, Staphylococcus aureus, MRSA and CONS, with the exception of Enterococcus species (against which either Penicillin or Vancomycin or Linezolid was individually effective), and Streptococcus species (against which either Penicillin, or Clindamycin was individually effective).
\end{abstract}

\section{Introduction}

Poorly controlled diabetes in a person can lead to the onset of a diabetic foot ulcer. Different micro-organisms, either alone or in combination, can be the causative agent(s), for the initiation of diabetic foot infections. Empirical therapy must be administered, by choosing appropriate antimicrobial agents, commensurate with the severity of infection, taking care to be guided by the prior knowledge of local data on bacterial epidemiology.

Tobalem and Uckay reported that diabetic foot infections could and would develop rapidly, and hence, would deserve a careful follow-up, after commencing empiric therapy regimen. Subsequently, however, a definitive antibiotic therapy must be decided, based on bacterial 
culture and sensitivity test results, in order to correctly target against the microbial causative agents. Generally, local baseline data on bacterial sensitivity can be considered as an effective tool in planning an empirical therapy. Early diagnosis and prompt antimicrobial treatment, when the infection remains mono-microbial, would help obtaining a cure.

\section{Objective of the Study}

The purpose of the study was to assess the feasibility of selecting appropriate antimicrobial agents (AMAs) for combating against mono-microbial infections, in respect of each pathogen, in the case of diabetic foot ulcer patients receiving treatment, in a tertiary care hospital, in PuducherryCity (South India), during the period of about ten months, from October 2015 to July 2016.

\section{Scope of the Study}

The results of the study can be used for correctly identifying antimicrobial agents (AMAs), in case of mono-microbial infections, in order to promptly arrange for an early treatment, and for preventing further aggravation. This trend is expected to offer some reliable guidance to hospitals located in rural and peripheral-urban settings, where patients belonging to the 'low-income' groups report themselves for treatment, on sensing any initial symptoms, and fearing/suspecting an infection.

\section{Materials and Methods}

The data on bacterial culture and antibiotic sensitivity patterns were obtained from the hospital records pertaining to the period of study, in the case of each diabetic foot ulcer patient. The data on antibiotic susceptibility of the various classes of antimicrobial agents was used in the statistical analysis (6)

\section{Results and Discussion}

The total number of clinical isolates collected from the 40-diabetic foot ulcer patients was 67. As presented in Figure-1, infection by a single pathogen (mono-microbial infection) was found in 18 patients (45.0\%); infection by two pathogens was $42.5 \%(17 / 40)$ and infection by three pathogens was $12.5 \%$ (5/40).

Table-1 presents the distribution of each pathogen, in the age groups of 30-49 years, 50-69 years, and 70-89 years, in the case of mono-microbial infections. It can be seen that the susceptible group of patients affected with mono-microbial infection among the diabetic foot ulcer patients was found to be maximum in the age-group of 50 to 69 , namely, $55.5 \%$ affecting 10 patients, followed by $27.75 \%$ affecting 5 patients in the age-group of 30-49 years, and $16.65 \%$ affecting 3 patients in the age-group of 70-89 years.

The mono-microbial infection pattern, as presented in Table-1, was as follows

5 numbers of Gram-positive organisms were involved in the mono-microbial infection, namely, Staphylococcus aureus, (22.2\%, infecting 4 patients), Methicillin-resistant Staphylococcus aureus (11.1\%,infecting 2 patients), Coagulase negative Staphylococcus (CONS) species (11.1\%,infecting 2 patients), Enterococcus species (5.5\%, infecting one person), and Streptococcus species (5.5\%, infecting one person).Mono-microbial infection by Gram-positive pathogens corresponded to $55.5 \%$.

7 numbers of Gram-negative organisms were involved in the mono-microbial infections, namely, Pseudomonas species (11.1\%, infecting 2 patients), Pseudomonas aeruginosa (5.5\%, infecting one person), Escherichia coli $(5.5 \%$, infecting one person), Atypical 
Escherichia coli (5.5\%, infecting one person),Klebsiellaoxytoca (5.5\%, infecting one person), Proteus mirabilis (5.5\%, infecting one person), and Proteus vulgaris (5.5\%,infecting one person).

Mono-microbialinfection by Gram-negative pathogens corresponded to $44.5 \%$ (out of which Enterobacteriaceae species corresponded to $27.75 \%$, ie., $62.3 \%$ of infection by Gram-negative pathogens), implying that Enterobacteriaceaespecies could play a predominant role in causing monomicrobial infections in diabetic foot ulcers, in contrast to Pseudomonas species (inclusive of Pseudomonas aeruginosa) which cause $16.65 \%$ of mono-microbial infections, in the present case

The maximum rate of mono-microbial infection occurred in the age-group of 50 to 69 years $(55.5 \%$, infecting 10 persons). Referring to Figure-2, it can be seen that the causative agents for mono-microbial infection were mostly Gram-positive pathogens (55.5\%), followed by Gram-negative pathogens $(44.5 \%)$. It must be noted that the Enterobacteriaceae species correspond to $27.75 \%$ (corresponding to $62.3 \%$ of the Gramnegative pathogens).

Infection by Pseudomonas species (including P.aeruginosa) was $16.65 \%$. Referring to Figure-3, it can be seen that the distribution of mono-microbial infection was $37.5 \%$ among the males, and $7.5 \%$ among the females, implying that diabetic foot infection was more prevalent in males than in females, due to the reality that males spend more time outdoors than females. Similarly, infection by two pathogens and three pathogens were lower among the females compared to males.

\section{Microbial Sensitivity Data}

In order to evaluate the feasibility of initiating empirical treatment for mono-microbial infections, in respect of each pathogen, the baseline data on microbial sensitivity available at the local centre were used.

The details of choices of appropriate AMAs (Anti Microbial Agents), in the case of each pathogen, are shown in Table-2, according to which the following trends were revealed:

i). Amikacin was found to be effective against Staphylococcus aureus, MRSA, and CONS, among the Gram-positive organisms.

ii). Wherever Amikacin failed, Linezolid, or Penicillin, or Vancomycin was found effective against Enterococcus species, and Penicillin or Clindamycin was effective against Streptococcus species.

iii). Amikacin was effective against all the Gram-negative organisms, excepting Proteus vulgaris against which Imipenem or Levofloxacin was found to be effective.

These choices of AMAs for combating against diabetic foot ulcer infections in the tertiary care hospital at Puducherry (South India) are agreeable to the findings of many other investigators who carried out similar studies in various other locations in South India, as cited in Table-2, column (v).

The AMAs to be tested in future studies against each pathogen are indicated in Table2 , in column (vi), based on the reports of other investigators who conducted studies in various other locations in South India. Information from the available literature suggests that Amikacin can be administered along with a 'matching class' of antibiotic, in order to avert the probable reduction in the efficacy of Amikacin, due to the production of antiaminoglycoside enzyme (initiated by the long term use of Amikacin by the patient). 
Prevalence of mono-microbial infections in various geographical locations in South India havebeen reported in varying percentages. In Kelambakkam near Chennai City (Tamil Nadu State), mono-microbial infection was reported as $50.0 \%$,with $34.8 \%$ of Gram-positive pathogens and65.2\% of Gram-negative pathogens, out of which $44.0 \%$ was Enterobacteriacea (11). In Chidambaram Town (Tamil Nadu State), it was reported as $23.7 \%$, with $5.8 \%$ of Gram-positive pathogens and $73.5 \%$ of Gram-negative pathogens, out of which $58.2 \%$ was Enterobacteriacea (20).

In Bengaluru (Kanataka State), it was reported as $100.0 \%$, with $24.4 \%$ of Gram-positive pathogens and $75.6 \%$ of Gram-negative pathogens, out of which $58.4 \%$ was Enterobacteriacea (21). In Thiruvananthapuram (Trivandrum, Kerala State), mono-microbial infection was reported as $41.7 \%$ (15).

In Manipal (near Mangalore, Karnataka State), it was reported as $67.0 \%$, with $38.0 \%$ of Gram-positive pathogens and $62.0 \%$ of Gramnegative pathogens, out of which $37.9 \%$ was Enterobacteriaceae (16). In Nellore town (Andhra Pradesh State), mono-microbial infection was reported as $81.2 \%$ (18).

In the present study, mono-microbial infection was $45.0 \%$, with $55.5 \%$ of Gram-positive pathogens, and $44.5 \%$ of Gram-negative pathogens, out of which $27.75 \%$ corresponded to Enterobacteriacae species, mostly agreeing with the results reported for many other locations in South India $(11,16,20,21)$.

The presence of each member of the Enterobacteriaceae species ( among the Gramnegative pathogens) could imply certain hardships in selecting a mono-drug therapy, as some of the Gram-negative pathogens could correspond to either ESBL-producers, or
AmpC beta-lactamase producers, or Carbapenemase-producers, or biofilmformers, because of which the bacterial resistance to antibiotic treatment would be exhibited in varying degrees.

Khan, et.al (22) reported that significant levels of resistance were exerted against Amikacin and other AMAs, by the Lactose-Fermenting Gram-negative Bacteria (LF-GNB), namely, Escherichia coli, Proteus species, Klebsiella species, Enterobacter species and Citrobacter species (in percentages varying from $16 \%$ to $45 \%$ of resistance to Amikacin), on par with Non-Lactose-Fermenting Gram-Negative Bacteria (Non-LF-GNB), namely, Pseudomonas aeruginosa and Acinetobacter species (which exerted resistances to Amikacin in percentages varying from $25 \%$ to $48 \%)$.

The results of the present study, in respect of effective antimicrobial agents, are fairly in agreement with the findings of Rapaka, A.R., et.al (23), according to which Amikacin was effective against the Gram-negative organisms, namely, Escherichia coli, Klebsiella species, and against Gram-positive organisms, namely, Staphylococcus aureusand Coagulase Negative Staphylococcus.

However, Amikacin faced resistance from Proteus species, in the present study. This implies that Gram-negative pathogens need a careful approach in the selection of antimicrobial agents, and that sensitivity of antimicrobial agents could vary from place to place.

Amikacinwas considered to be a better choice for combating against Escherichia coli, Proteus species, and Klebsiella species, 'in case of moderate' grade of foot infections (24).In general, Aminoglycosides, used in treating diabetic foot infections, correspond to $21.46 \%$, whereas, Quinolones correspond to 
20.2\%, and Cephalosporins correspond to $19.44 \%$ (25). Most of the diabetic foot ulcer patients happen to suffer from co-morbid conditions that require analgesic drugs during the treatment for curing diabetic foot ulcers $(25,26)$. This must be taken into account while choosing thetherapy regimen.

There was a general agreement between the bacterial species prevalent in the monomicrobial flora observed in the present study and the findings of Priyadarshini, S., et.al (11), in the sense that many Gram-negative species were individually present in the monomicrobial diabetic foot infections, whereas many investigators in foreign countries indicated that mono-microbial diabetic foot infections were mostly caused by Grampositive pathogens, namely, Staphylococcus aureus, Streptococcus species, etc $(27,27 \mathrm{~b}, 28)$.

The management of diabetic foot ulcers cannot fully rely upon antimicrobial treatment alone. It must be combined with concurrent debridement procedures and wound-dressing arrangements $(29,8,30)$. The role of a microbiologist or an expert on infectious diseases must be made available at several stages of the treatment period, to enable the clinician to take judicious decisions, in the choice of antibiotic therapy, at his/her exclusive discretion.

However the baseline data on the culture-cumsensitivity available at the local centre would be of great value, as a reference document. Trials of appropriate dosages of Ceftriaxone plus Ciprofloxacin versus Amikacin plus Ceftazidime, have been suggested by some investigators ( 31,32 ).Peter, N., et.al (33) indicated Amikacin plus Cefotaxime as a dual therapy, and Amikacin plus Metronidazole plus Amoxicillin/clavulanic acid by way of triple therapy, probably, in the case of polymicrobial diabetic foot infections. By way of mono-therapy, Ciprofloxacin and
Metronidazole were indicated.

Ramirez and Tolmasky (34) reported that Amikacin, as an aminoglycoside, could deserve the status of an essential component of armamentarium against serious infections caused by Gram-negative and Gram-positive bacterial species, and that Amikacin could be used always along with another class of antibiotic (in such a way that it would not involve any antagonistic side effect).

The efficacy of Amikacin could get reduced due to the production of aminoglycosidemodifying enzymes, particularly, AAC(6')-1b, and this is the reason thatAmicacin is administered always in combination with one more antibiotic of another 'matching' class. It was reported that un-modified therapy by Imipenem was as effective as a combination therapy by Ceftazidime/Amikacin in clinically and 'bacteriologically documented' infections (35).

A combination therapy with Amikacin and Piperacillin/tazobactam, or Imipenem and Vancomycin was reported as successful, in the treatment for diabetic foot infections (36). Ali \& Al-Shabaki (37) reported that Amikacin was susceptible to all the bacterial species, namely, Staphylococusaureus, Pseudomonas species, Escherichia coli, Klebsiella species, etc.,in percentages higher than $66.7 \%$, excepting Pseuodomonasaeruginosa and Proteus mirabilis. This is in agreement with the findings of the present study..

The treatment approach would include procedures such as wound-cleaning, debridement, wound-dressings, antibioticimpregnated dressings, and antibiotic treatment, based on clinical trial results $(38$, $39,40,4)$. 
Table.1 Age Group-wise Distribution of Mono-microbial Infection

\begin{tabular}{|c|c|c|c|c|c|}
\hline S,no. & Pathogen & $\begin{array}{c}\text { 30-49 } \\
\text { Yrs. }\end{array}$ & $\begin{array}{c}\text { 50-69 } \\
\text { Yrs. }\end{array}$ & $\begin{array}{c}\text { 70-89 } \\
\text { Yrs. }\end{array}$ & Total \\
\hline 1. & S.aureus & $\ldots$ & $16.65(3)^{*}$ & $5.55(1)^{*}$ & 22.20 \\
\hline 2. & $* *$ MRSA & $5.55(1)$ & $5.55(1)$ & $\ldots$ & 11.10 \\
\hline 3. & $+\mathrm{CONS}$ & $\ldots$ & $5.55(1)$ & $5.55(1)$ & 11.10 \\
\hline 4. & Enterococcus spp. & $5.55(1)$ & $\ldots$ & $\ldots$ & 5.55 \\
\hline 5. & Streptococcus spp. & $\ldots$ & $\ldots$ & $5.55(1)$ & 5.55 \\
\hline 6. & $\begin{array}{l}\text { Beta-hemolytic } \\
\text { Streptococcus spp. }\end{array}$ & $\ldots$ & $\ldots$ & $\cdots$ & $\cdots$ \\
\hline 7. & Pseudomonas spp. & $5.55(1)$ & $5.55(1)$ & $\ldots$ & 11.10 \\
\hline 8. & P.aeruginosa & $5.55(1)$ & $\ldots$ & $\ldots$ & 5.55 \\
\hline 9. & Enterobacter spp. & $\ldots$ & $\ldots$ & $\ldots$ & $\cdots$ \\
\hline 10. & E.coli & $5.55(1)$ & $\ldots$ & $\ldots$ & 5.55 \\
\hline 11. & Atypical E.coli & $\ldots$ & $5.55(1)$ & $\ldots$ & 5.55 \\
\hline 12. & ESBL-producing E.coli & $\cdots$ & $\cdots$ & $\cdots$ & $\cdots$ \\
\hline 13. & K.pneumoniae & $\ldots$ & $\ldots$ & $\cdots$ & $\ldots$ \\
\hline 14. & K.oxytoca & $\ldots$ & $5.55(1)$ & $\ldots$ & 5.55 \\
\hline 15. & Klebsiella spp. & $\ldots$ & $\ldots$ & $\ldots$ & $\cdots$ \\
\hline 16. & P.mirabilis & $\cdots$ & $5.55(1)$ & $\ldots$ & 5.55 \\
\hline 17. & P.vulgaris & $\ldots$ & $5.55(1)$ & $\ldots$ & 5.55 \\
\hline 18. & Proteus spp. & $\cdots$ & $\cdots$ & $\ldots$ & $\cdots$ \\
\hline \multirow[t]{2}{*}{19.} & Citrobacter spp. & $\ldots$ & $\ldots$ & $\ldots$ & $\ldots$ \\
\hline & Total & $27.75(5)$ & $55.50(10)$ & $16.65(3)$ & $\sim 100.0$ \\
\hline
\end{tabular}

**Note : Numbers within parentheses indicate the number of patients infected with a single, specific pathogen

** MRSA = Methicillin Resistant Staphylococcus aureus $) ;+$ CONS $=($ Coagulase

Negative Staphylococcus 
Table.2 AMAs selected against mono-microbial infections

\begin{tabular}{|c|c|c|c|c|c|}
\hline S.No & $\begin{array}{l}\text { Pathogen } \\
\text { (ii) }\end{array}$ & $\begin{array}{l}\text { No. of Cases } \\
\text { (iii) }\end{array}$ & $\begin{array}{c}\text { AMAs } \\
\text { Sensitive } \\
\text { (iv) }\end{array}$ & $\begin{array}{c}\text { AMAs } \\
\text { Of choice } \\
\text { (references) } \\
\text { (v) }\end{array}$ & $\begin{array}{c}++ \\
\text { Other AMAs } \\
\text { To be Tested } \\
(\text { vi) }\end{array}$ \\
\hline $\begin{array}{l}\mathbf{G}(+) \\
1 .\end{array}$ & S.aureus & $3 \mathrm{M}, 1 \mathrm{~F}$ & $\begin{array}{l}\text { Ak,Cd, } \\
\text { V,Lin }\end{array}$ & $\operatorname{Ak}(7,8,9,10)$ & $\begin{array}{l}\text { Amc,Cip,At,E,Dox, Cpm,E,G,Cfxtn, } \\
\text { Tet,Dox,Oxa,Tmp/Smx,Cd, Cfzln }\end{array}$ \\
\hline 2. & $M R S A$ & $2 \mathrm{M}$ & $\mathrm{Ak}, \mathrm{V}$ & $\operatorname{Ak}(8,11)$ & At,Cd, Lin,Dox Tmp/Smx \\
\hline 3. & CONS & $2 \mathrm{M}$ & $\mathrm{Ak}, \mathrm{Cd}$ & $\operatorname{Ak}(7,12,13)$ & P,E,Lin, Ctr,Tet \\
\hline 4. & Enterococcus & $1 \mathrm{M}$ & $P, V$ & $\operatorname{Lin}(14,15,13)$ & Amp,Amc,Clrm, Dox,G,E, I,Tet,Cip \\
\hline 5. & Streptococcus sp. & $1 \mathrm{~F}$ & $\mathrm{P}, \mathrm{Cd}$ & $\mathrm{P}(9,11)$ & Amp,At,E,Ctr,V, Cot,G,Teic,Rif, Amox \\
\hline \multicolumn{6}{|l|}{$\mathbf{G}(-)$} \\
\hline 6. & Pseudomonas sp. & $2 \mathrm{M}$ & Ak,Pit & $\operatorname{Ak}(7,11,13)$ & G,Cpm,Caz \\
\hline 7. & P.aeruginosa & $1 \mathrm{M}$ & Ak, Pit, & $\operatorname{Ak}(8,16)$ & $\begin{array}{c}\text { At,G,Cip,Cfs,Caz,I,Cpm,Cfxtm,Cfrxm,Ntlm } \\
\text { cn,Pi,Tob, Tet,Tcc,Cot,Lin }\end{array}$ \\
\hline 8. & E.coli & $1 \mathrm{M}$ & $\mathrm{Ak}, \mathrm{I}$ & $\operatorname{Ak}(7,8,11,13,17,18)$ & G,Cfs,Pit,Tcc \\
\hline 9. & Atypical & $1 \mathrm{M}$ & Ak & Ak (not isolated & G,I,Cfs, Pit,Tcc \\
\hline 10. & K.oxytoca & $1 \mathrm{~F}$ & Ak & $\operatorname{Ak}(9,11,13)$ & Cot,Clrm,Cftxm,G, Pit,I,Tet \\
\hline 11. & P.mirabilis & $1 \mathrm{~F}$ & Ak,Pit & $\operatorname{Ak}(12,13,18)$ & G,I,Caz,Tet,Cip,Ctr,Cpm,Cftxm, \\
\hline \multirow[t]{2}{*}{12.} & P.vulgaris & $1 \mathrm{M}$ & I, Lev & I $(11,12,13,17,18,19)$ & ,G,Pit,Cip Cpm,Cftxm,Tet, Caz.Ctr,Ntlmcn, \\
\hline & Total & 18 & & & \\
\hline
\end{tabular}

++ Note: Additional AMAs to be included in testing the susceptibility; as per clues indicated by other investigators in South India.

$\mathrm{Ak}=$ Amikacin; Amox $=$ Amoxicillin; Amc $=$ Amoxicillin/clavulanic acid; Amp=Ampicillin; At=Aztreonam; $\mathrm{Cd}=\mathrm{Clindamycin} ;$ Cip : Ciprofloxacin; $\mathrm{Clrm}=$ Chloramphenicol; Caz= Ceftazidime; $\mathrm{Cftxm}=$ Cefotaxime; $\mathrm{Cfrxm}=$ Cefuroxime; Cftxn= Cefoxitin; Cfzln= Cefazolin; Cflxn= Cefalexin; Cfs= Cefaperazone/sulbactam; Cpm= Cefepime; Cot= Cotrimoxazole; Ctr=Ceftriaxone; Dox :Doxicycline; E=Erythromycin; G : Gentamicin; I=Imipenem;

Lin=Linezolid; Lev = Levofloxacin; Ntlmen= Netilmycin; Oxa= Oxacillin; P : Penicillin; Pi= Piperacillin; Pit :Piperacillin/tazobactam; Rif=Rifampicin; Teic= Teicoplanin; Tet=Tetracycline;Tcc= Tetracycline/clavulanicacid; Tob= Tobramycin; Tmp/smx= Trimethoprim/sulfamethoxazole; V= Vancomycin . 
Int.J.Curr.Microbiol.App.Sci (2020) 9(1): 1416-1426

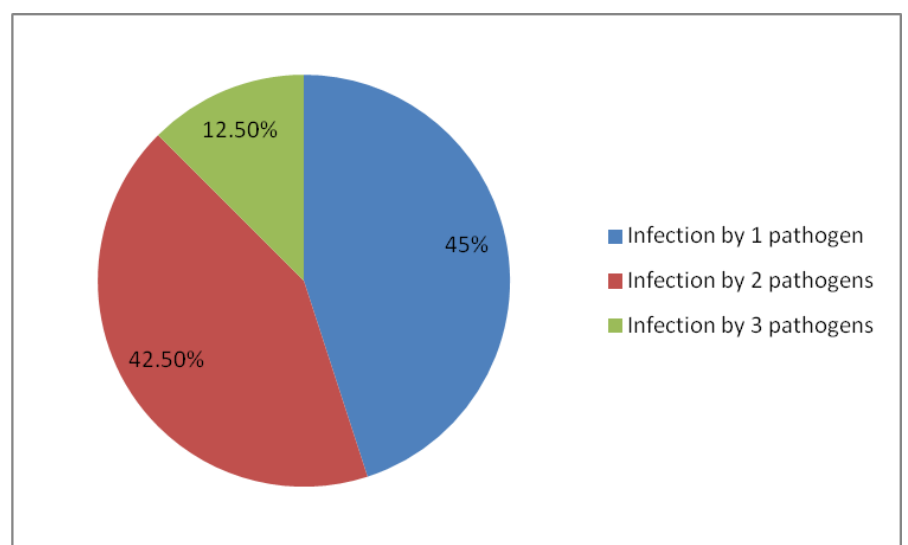

Fig.1 Infection pattern in a single patient

Gram - Positive
(55.5\%)
Gram -
Negative(44.5\%)

Fig.2 Distribution of Bacterial Pathogens in Mono-microbial Infection

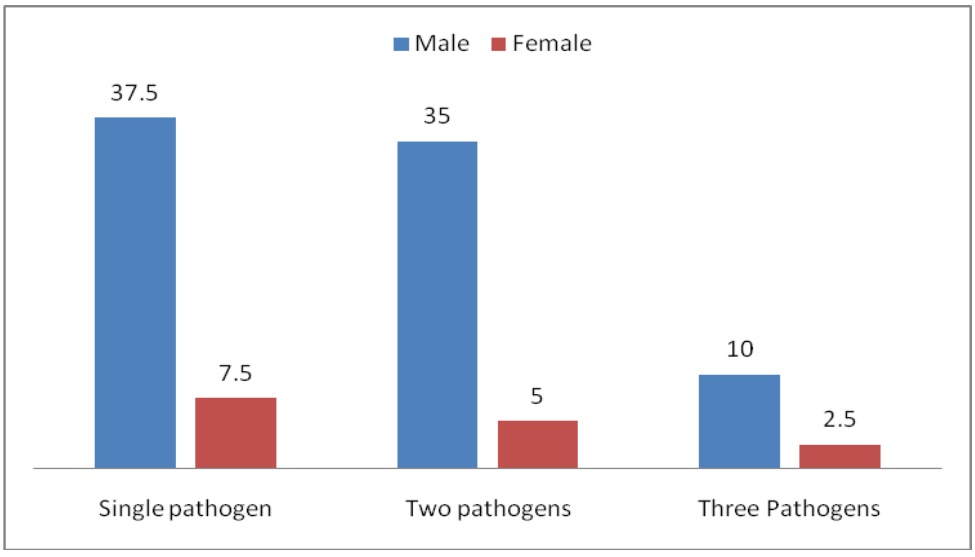

Fig.3 Infection rate among males versus females 
In case of mono-microbial infections, Amikacin was found to be a promising antimicrobial agent, in treating diabetic foot infections, in the case of mono-microbial infections, against some Gram-positive pathogens such as Staphylococcus aureus, MRSA, and CONS. Amikacin faced resistance from Enterococcus species and Streptococcus species.

Amikacin was also found to be effective against some Gram-negative organisms such as Pseudomonas species, Pseudomonas aeruginosa, Escherichia coli, Atypical Escherichia coli, and Klebsiellaoxytoca.

Amikacin faced resistance from Proteus vulgaris. The best approach in treating monomicrobial infection of diabetic foot ulcers will be to select the empiric therapy based on the local baseline data, and arrange for early identification of the causative pathogen, and to select the confirmatory therapy as guided by bacterial sensitivity results.

Additional AMAs identified in Table-2 (column vi) will be helpful for future studies toward strengthening the data-bank on bacterial sensitivity at the local centre,

\section{References}

Ali, J.F., and Al-Shabaki, 2014, "Bacteriological Evaluation of Diabetic Foot Infections of Patients in Mosul City (Iraq) and Antibiotic Sensitivity Pattern", International Journal of Advanced Research, 2(5) : 614-623.

Anandi, C., Alaguraja, D., Natarajan, V., Ramanathan, M., Subramaniam, C.S., et.al, 2004, "Bacteriology of Diabetic Foot Lesions", Indian J. Med. Microbiol., $22: 175-178$

Anorshirvani, A.A., Zarinfar, N., Rafiee, M., 20 August 2018, "Effect of Combination Therapy of Ceftazidime/Amikacin and Monotherapy with Imipenem on the
Treatment of Fever and Neutropenia in Patients with Cancers", Open Access Maced J. Med. Sci., 6(8) : 1423-1430.

Ariffin, H., et.al,2001, "Single-daily ceftriaxone plus amikacin versus thrice-daily ceftazidime plus amikacin as empirical treatment of febrile neutropenia in children with cancer", Randomized Controlled Trial, J. Paediatric Child Health, 2001.

Banashankari, G., Rudresh, H., and Harsha, A., 2012, "Prevalence of Gram-negative bacteria in diabetic foot - a clinicomicrobiological study", Al Ameen Journal of Medial Sciences, 5(3) : 224232.

Banu, A., Hassan, M.M.N., Rajkumar, J., and Khan, K.A., 2015, "Prospective Study of Multidrug Resistant Bacteria Causing Diabetic Foot Ulcers in South India", Journal of Science, 5(8) : 626-629.

Benwan, K.A., Mulla, A.A.,Rotimi, V.O., 2012, "A study of the microbiology of diabetic foot infections in teaching hospital in Kuwait", Journal of Infection and Public Health, 5(1) : 1-8.

Davis, S.C., Martinez, L., Kirsner, R., 2006, "The Diabetic Foot: The Importance of Biofilms and Wound Bed Preparation", Curr. Diab. Rep., 6(6) : 439-45.

Elahe Ellhami, et.al, 2016, "Prescribing Pattern of Different Antibiotics and Analgesics Used in Patients with Diabetic Foot Ulcer", Indo American Journal of Pharmaceutical Research, 6(01) : 39873995.

Gupta, S., 2012, "Management of Diabetic Foot", Medicine Update, 22 : 287-293.

Jenifer, J., Sekkizhar, G., Kumpatla, S., Viswanathan, V., 2013, "Bioburden Vs Antibiogram of Diabetic Foot Infection", Clin. Res. On Foot \& Ankle, 1:121. (Vol.1, Issue 3; 1000121).

Jude, E.B., and Unsworth, P.F., 2004, "Optimal Treatment of Infected Diabetic Foot Ulcers", 21(13) : 883-50.

Junior Sundaresh, N., Narendran, S., Ramesh, R., Kesarijagadeesan, R., and Nasara Reddy, N., 2014, "Clinical and 
Microbiological Study of Diabetic Foot in Patients Admitted at RMMCH, Chidambaram, Tamil Nadu, India, Journal of Pharma. \&Sci.Innovation, (2):153-138

Kandati, J., Pathapati, RM,Buchineni, M., Sateesh,S., Premanadam, N.,2015, "Microbiological profile of diabetic foot ulcer infections in a tertiary care hospital in South India-Aprospective study", International journal of Science and applied Research, 2(1) : 64-70.

Kavitha, Y., and KhajaMohiddin, S., Sep 2014, "Bacteriological profile of diabetic foot infections in a tertiary care teaching hospital", Indian Journal of Basic and Applied Medical Research, 3(4) : 260266.

Khan, D.M., Moosabba, M.S., VenkatakrishnaRao, I., 2016, "Prevalence of Diabetic Foot Ulcer Infections associated with Gram-Negative Bacteria with Special Reference to Drug Resistant Isolates", nternational Journal of Biomedical Research, 711) : 765-770.

Leela Rani, K., Madhumathy, Belwadi, S., and Ramana, B.V., 2013, "Bacterial Profile of Diabetic Foot Ulcer", International Journal of Pharmaceutical Research and Bioscience, 22(2) : 36-45. ISSN : 22778713.

Lipsky, B.A., 2007, "Empirical therapy for diabetic foot infections : are there clinical clues to guide antibiotic selection?", Clinical Microbiol. Infect., 13 : 351-353.

Lipsky, B.A., et.al., "2012 Infectious Society of America Clinical Practice Guidelines for the Diagnosis and Treatment of Diabetic Foot Infections", Clin. Infect. Dis., 54(12): 132-157.

Little, J.R., Levin, M.E., O’Neal, L.W., Mosby, C.O., "Bacteriology and the diabetic foot", In Levin JR and O'Neil ME, Editors, The Diabetic Foot, St.Louis : Mosby; 1973; pp. 86-96.

Manikandan, C., Prabakaran, P.,2015, "Clinical and Bacteriological Profile of Diabetic Foot in Pattukkottai Area Hospitals", Int.
J. Curr. Res. Acad. Review, 3(4); 166173.

Manisha, A., Brandao, D.,2013, "Guidelines For Treatment of Patients with Diabetes and Infected Ulcers", J.Cardiovasc.Surg., (Torino), $54: 193-200$.

Mathangi, T., and Prabhakaran, P., "Prevalence of Bacteria Isolated from Type-2 Diabetic Foot Ulcers and the Antibiotic Susceptibility Pattern", 2013, Int. J. Curr.Microbiol. App. Sci., 2(10) :x-xx.

Meenakshi sundaram, C., Uma Rani, J., UshaAnadRao, Mohan, V., and Vasudevan, V., 2017, "Hypothetical Estimate of Drug Burden on Diabetic Foot Ulcer Patient and Its relevance to Microbiological Analysis", International Journal of Current Microbiology and Applied Sciences, 6(6): 1139-1159. (https:www.researchgate.net/publication/ 317610450_Hypothetical_Estimate_of_D rug_Burden).

Meenakshisundaram, C.,G.Jayalakshmi, P.Selvaraj, 3018, "Diabetic Foot Ulcer : A study in a Tertiary Care Hospital in Puduchery (South India)", ParipexIndian Journal of Research, 7(7) : 23-27.

Metallidis, S., et.al, 2008 Dec, "A prospective, controlled, randomized, non-blind, comparative study of the efficacy and safety of a once daily high dose of ceftriaxone plus ciprofloxacin versus thrice daily ceftazidime plus amikacin in empirical therapy of febrile neuropenic patients", Randomised Controlled Trial, Eur. J. Intern. Med., 19(8):619-24.

Mukkanath, S.N., Manjunath, R., Desai, M., 2015, "A Study of the Bacteriological Profile of Diabetic Foot Ulcer and antibiotic Sensitivity Pattern", J. of Evolution of Medicine ad Dent. Sci., $4(39): 683240$.

Murali,T.S., Kavitha, S., Spoorthi,J.,Bhat,D., Bharath Prasad, A.S., Upton, Z., Ramachandra,L., Acharya,R.V., and Satyamoorthy, k., 2014, "Characteristics of microbial drug resistance and its correlates with diabetic foot infections", 64 : 1377-1385. 
Nair, S.R., Rajan, R., Saradadevi, KarunakaranLaithaBai, 2015, "A clinicmicrobiological study of diabetic foot ulcers from South Kerala", Journal of the Academy of Microbiology, 17(2) : 94-99.

Peter, N., Cherian, N., Thomas, S., George, S., Jr.Sundaresh, N., 2017, "Study of Prescribing Pattern and Use of Antibiotic in the Management of Wound Infections", Asian Journal of Pharmaceutical and Clinical Research, 10(2) : 210-213.

Phillips, P.L., Wolcott, R.D., Fletcher, J., et.al, 2010, "Bio-films made easy", Wounds International, 1(3) : Available at http : //www.woundinternational.com, assessed March 2013.

Priyadarshini S., Jaya, M., and Linda Susan,M, 2013, "The Bacteriology of Diabtic Foot Ulcers, with a Special Reference to Multi Drug Resistant Strains", J. Clin. Diagn. Res.,7 (3) : 441-445.

Ramirez, and Tolmasky, M., 2017, "Amikacin :Uses , Resistance, and prospects for Inhibition", Molecules, 22:2267. (doi:10.3390/molecules 22122267).

Rapaka, A.R., Veeramalla, S., Gollamandala, M.,Maloth, R.N., Prabhumithra, S., Adla, N., Gade, A., Bandaru, S., and Puchchakayala, G., 2016, "A Prospective Observational Study, of Microbiological Analysis and Antibiotic Sensitivity Patterns in Diabetic Foot Ulcer Patients", Advances in Biological Research, 10(4) : 248-256.

Sekhar, S.M.,Vyas, N., Unnikrishnan, M.K., Mukhopadhyay, C., 2015, "Antimicrobial Susceptiiblity Pattern in diabetic foot ulcer : A case study ", Ann. Med. Health Sci. Res., $4: 2$ : 742-5.

Spichler, A., Hurwitz, B.L., Armstrong, D.G., Lipsky, B.A., 2015, "Microbiology of diabetic foot infections : from Louis Pasteur to "Crime Scene Investigation", BMCMed., 13(2): 1-13. (PMID: 25564342).

Suganthi, P., Prasanth, D.A.,2014, "Bacteriological Profile of Diabertic Foot Infections", International Journal of Innovative Research in Science,3(7) :14688-'92.PMID : 14611743.

Suresh, A., Muthu, G., Srivani, R., and Moses, A., 2011, "Aerobic bacterial Resistance in Diabetic Foot Ulcer from Chennai", International journal of Pharma. and Bio. Sciences, 2(2): B-517 to B-522.

The Health Line Editorial Team, 2016, "Diabetic Foot Pain and Ulacers : Causes and Treatment", medically reviewed by Steve Kim, M.D., Feb.18, 2016.

Tobalem , M., and Uckay, I.,2013, "Evolution of Diabetic Foot Infections", N.Engl.J. Med., 2013, Dec. 5, 369:2252.

Umadevi, S., Kumar, S., Joseph, N.M., Easow, J.M., Kandhakumari, G., Srirangaraj, S., Raj, S., Stephen, S., 2011, "Microbiology of Diabetic Foot Infections", Indian Journal of Medical specialities, 2910 ; 1217.

Wounds International, 2013, International Best Practice Guidelines : Wound Management in Diabetic Foot Ulcers", Available from www.woundsinternational.com

\section{How to cite this article:}

Meenakshi Sundaram, C., G. Jayalakshmi and Selvaraj. P. 2020. Mono-microbial diabetic footinfections: Feasibility for prompt treatment. Int.J.Curr.Microbiol.App.Sci. 9(01): 14161426. doi: https://doi.org/10.20546/ijcmas.2020.901.157 